\title{
O treino muscular inspiratório aumenta a deposição de radioaerossol pulmonar em pacientes com DPOC com fraqueza muscular respiratória?: Um protocolo de estudo para ensaio clínico randomizado
}

\author{
Does inspiratory muscle training increase pulmonary radioaerosol deposition in COPD \\ patients with respiratory muscle weakness?: A study protocol for a randomized clinical trial \\ ¿El entrenamiento de los músculos inspiratorios aumenta el depósito de radioaerosol \\ pulmonar en pacientes con EPOC y debilidad de los músculos respiratorios?: Protocolo de \\ estudio para ensayo clínico aleatorizado
}

Recebido: 07/02/2022 | Revisado: 14/02/2022 | Aceito: 16/02/2022 | Publicado: 25/02/2022

\author{
Lays Rodrigues \\ ORCID: https://orcid.org/0000-0002-2359-1363 \\ Universidade Federal de Pernambuco, Brasil \\ E-mail: laysdasilva@hotmail.com \\ Dulciane Paiva \\ ORCID: https://orcid.org/0000-0001-5629-3285 \\ Universidade Federal de Pernambuco, Brasil \\ E-mail: dulciane@unisc.br \\ Taciano Rocha \\ ORCID: https://orcid.org/0000-0001-6190-1425 \\ Universidade Federal de Pernambuco, Brasil \\ E-mail: taciano_rocha@hotmail.com \\ Daniella Cunha Brandão \\ ORCID: https://orcid.org/0000-0001-8805-6815 \\ Universidade Federal de Pernambuco, Brasil \\ E-mail: daniellacunha@hotmail.com \\ Armele Dornelas de Andrade \\ ORCID: https://orcid.org/0000-0001-9430-4395 \\ Universidade Federal de Pernambuco, Brasil \\ E-mail: armeledornelas@hotmail.com
}

\begin{abstract}
Resumo
A doença pulmonar obstrutiva crônica (DPOC) caracteriza-se por limitação crônica do fluxo aéreo, em muitos casos podendo ocorrer também disfunção muscular respiratória associada e déficit na capacidade de gerar força contrátil, resultando em fraqueza dessa musculatura. O objetivo desse estudo foi avaliar a eficácia do Treinamento Muscular Inspiratório (TMI) sobre a deposição de radioaerossol pulmonar em pacientes com DPOC e fraqueza muscular respiratória. Trata-se de um ensaio clínico randomizado e duplo cego que submeterá pacientes com DPOC de diferentes estadiamentos segundo os critérios da Global Initiative for Chronic Obstructive Lung Disease (GOLD) e com fraqueza muscular respiratória $\left(\mathrm{PI}_{\max }<60 \mathrm{cmH}_{2} \mathrm{O}\right)$ ao TMI (Grupo TMI) e ao treino com carga sub-terapêutica (Grupo Controle). Os pacientes do Grupo TMI iniciarão o treinamento com $60 \%$ da $\mathrm{PI}_{\max }$ e a intensidade do treino será aumentada semanalmente em $50 \%$ sob os novos valores de $\mathrm{PI}_{\max }$ mensurados. $\mathrm{O}$ grupo controle realizará o protocolo de com $10 \%$ da $\mathrm{PI}_{\max }$ inicial e esse valor se manterá constante ao longo do treinamento. A deposição pulmonar de radioaerossol será mensurada através da cintilografia pelo índice de deposição pulmonar (IDP), obtido através da razão entre a quantidade de contagens de cada região de interesse (ROI) pela quantidade total de contagens do respectivo pulmão. Há uma escassez na literatura sobre o comportamento da distribuição pulmonar de radioaerossóis nessa população, até o momento, não foram encontrados estudos que estabeleçam relação entre o TMI em pacientes com DPOC e fraqueza muscular respiratória e a melhora da deposição de radioaerossol pulmonar. Tal constatação reafirma a importância do desenvolvimento do presente estudo que poderá contribuir para uma melhor perspectiva dos pacientes com DPOC.
\end{abstract}

Palavras-chave: DPOC; Exercício respiratório; Terapia com aerossóis; Cintilografia.

\footnotetext{
Abstract

Chronic obstructive pulmonary disease (COPD) is characterized by chronic airflow limitation, in many cases there may also be associated respiratory muscle dysfunction and a deficit in the ability to generate contractile
} 
force, resulting in muscle weakness. The aim of this study was to evaluate the effectiveness of Inspiratory Muscle Training (IMT) on pulmonary radioaerosol deposition in patients with COPD and respiratory muscle weakness. This is a randomized, double-blind clinical trial that will submit patients with COPD of different stages according to the criteria of the Global Initiative for Chronic Obstructive Lung Disease (GOLD) and with respiratory muscle weakness (MIP < 60cmH2O) to IMT (IMT Group) and sub-therapeutic load training (Control Group). Patients in the IMT Group will start training at $60 \%$ of MIP and training intensity will be increased weekly by $50 \%$ under the new MIP values measured. The control group will perform the protocol with $10 \%$ of the initial MIP and this value will remain constant throughout the training. The pulmonary deposition of radioaerosol will be measured through scintigraphy by the pulmonary deposition index (PDI), obtained through the ratio between the number of counts in each region of interest (ROI) by the total number of counts in the respective lung. There is a scarcity in the literature on the behavior of the pulmonary distribution of radioaerosols in this population, so far, no studies have been found that establish a relationship between the IMT in patients with COPD and respiratory muscle weakness and the improvement of pulmonary radioaerosol deposition. This finding reaffirms the importance of developing the present study, which may contribute to a better perspective of patients with COPD. Keywords: COPD; Breathing exercise; Aerosol therapy; Scintigraphy.

\section{Resumen}

La enfermedad pulmonar obstructiva crónica (EPOC) se caracteriza por una limitación crónica al flujo aéreo, en muchos casos también puede asociarse una disfunción de los músculos respiratorios y un déficit en la capacidad de generar fuerza contráctil, lo que resulta en debilidad muscular. El objetivo de este estudio fue $\boldsymbol{e}$ valuar la efectividad del entrenamiento muscular inspiratorio (EMI) sobre el depósito de radioaerosol pulmonar en pacientes con EPOC y debilidad de los músculos respiratorios. Se trata de un ensayo clínico aleatorizado, doble ciego, que someterá a EMI a pacientes con EPOC en diferentes estadios según los criterios de la Global Initiative for Chronic Obstructive Lung Disease (GOLD) y con debilidad de los músculos respiratorios ( $\mathrm{PI}_{\max }<60 \mathrm{cmH} 2 \mathrm{O}$ ) (Grupo EMI) y entrenamiento con carga subterapéutica (Grupo Control). Los pacientes del Grupo EMI comenzarán a entrenar al $60 \%$ de la $\mathrm{PI}_{\max }$ y la intensidad del entrenamiento se incrementará semanalmente en un $50 \%$ por debajo de los nuevos valores de la $\mathrm{PI}_{\max }$ medidos.El grupo control realizará el protocolo con el 10\% de la $\mathrm{PI}_{\max }$ inicial y este valor se mantendrá constante durante todo el entrenamiento. El depósito pulmonar de radioaerosol será medido mediante gammagrafía por el índice de depósito pulmonar (IDP), obtenido a través de la relación entre el número de conteos en cada región de interés (ROI) por el número total de conteos en el respectivo pulmón. Existe escasez en la literatura sobre el comportamiento de la distribución pulmonar de los radioaerosoles en esta población, hasta el momento no se han encontrado estudios que establezcan una relación entre EMI en pacientes con EPOC y debilidad de los músculos respiratorios y la mejoría de la pulmonar. depósito de radioaerosoles. Este hallazgo reafirma la importancia de desarrollar el presente estudio, que puede contribuir a una mejor perspectiva de los pacientes con EPOC.

Palabras clave: EPOC; Ejercicio de respiración; Aerosolterapia; Gammagrafía.

\section{Introdução}

A doença pulmonar obstrutiva crônica (DPOC) trata-se de uma patologia caracterizada principalmente por limitação do fluxo aéreo, geralmente progressivo e parcialmente reversível (Petrovic et al.,2012; Global Strategy for the Diagnosis, Management, and Prevention of Chronic Obstructive Lung Disease, 2017). A fraqueza muscular respiratória, definida como um déficit recorrente no cumprimento da função muscular e na sua capacidade de gerar pressões se constitui em um achado de elevada prevalência nos pacientes com DPOC (Martínez-Llorens et al., 2011; Vilaró et al., 2010). Ela é observada em todos os graus de severidade da doença, entretanto é mais comum em indivíduos classificados como graves (Barreiro, et al.,2015). O diafragma, principal musculo inspiratório, é um dos mais afetados, sendo esta condição mais pronunciada na presença de hiperinsuflação (Polkey et al.,1996) o que leva esses pacientes a uma desvantagem mecânica que pode estar associada à fraqueza da musculatura respiratória. Esse déficit pode gerar um subsequente aumento da sensação de esforço respiratório, também conhecia como dispneia (Killian et al., 1988) sendo esse um dos sintomas mais limitantes dessa doença (Hamilton et al.,1995) com impacto direto sobre a qualidade de vida (QV) e capacidade de desenvolvimento das atividades de vida diária (AVD) desses indivíduos (Hu et al., 2005; Gruenberger et al., 2017), com repercussão negativa também no que diz respeito à produtividade no ambiente de trabalho (DiBonaventura et al.,2012). 
A reabilitação pulmonar é uma terapêutica eficaz e capaz de produzir melhorias significativas nessa população (McCarthy et al., 2015). Nesse contexto encontra-se o treinamento muscular inspiratório (TMI) (Gosselink et al., 2011), que poderia produzir uma melhora na mecânica respiratória (Leith et al., 1976) sendo capaz de aumentar a $\mathrm{PI}_{\max }$, o desempenho muscular (Petrovic et al., 2012) a qualidade de vida e reduzir a sensação de dispneia em pacientes com DPOC (Geddes et al., 2008) em todos os graus de severidade da doença (Global Strategy for the Diagnosis, Management, and Prevention of Chronic Obstructive Lung Disease, 2017). Os primeiros estudos que evidenciam o benefício do uso do TMI em pacientes com DPOC datam da década de 80 (Chen et al., 1985; Goldstein et al., 1989). Esses efeitos também são observados a longo prazo, em que se evidencia diminuição do uso dos serviços de saúde e dos dias de internação hospitalar na mesma população (Beckerman et al., 2005).

Uma vez que a força muscular respiratória tem influência direta na capacidade de gerar volumes pulmonares (Lanza et al., 2013), o fortalecimento dessa musculatura poderia também além dos benefícios já conhecidos, otimizar a deposição de aerossóis e por consequência a terapêutica inalatória desses pacientes que devido ao prejuízo da função pulmonar, são muitas vezes são incapazes de gerar uma inspiração profunda (Wieshammer et al., 2008). A avaliação da distribuição de radioaerossol pulmonar já se mostrou possível em alguns estudos (Kwok et al., 2019; Alcoforado et al., 2017), mas até o momento não foram encontrados estudos na população de pacientes com DPOC. Dessa forma, o objetivo do presente estudo é avaliar o efeito do treino muscular inspiratório sobre a deposição de radioaerossol pulmonar em pacientes com doença pulmonar obstrutiva crônica com fraqueza muscular respiratória e o impacto que essa intervenção pode trazer em aspectos como nível de dispneia e qualidade de vida desses indivíduos.

\section{Metodologia}

O estudo será desenvolvido no Laboratório de Fisioterapia Cardiopulmonar (LACAP), no Departamento de Fisioterapia (DeFisio) da Universidade Federal de Pernambuco (UFPE) e no serviço de Medicina Nuclear do Hospital das Clínicas (HC). O projeto de pesquisa foi aprovado pelo comitê de ética em pesquisa em seres humanos da Universidade Federal de Pernambuco sob o número de protocolo CAAE: 03040918.8.0000.8807.

A população do estudo será composta por pacientes com diagnóstico de doença pulmonar obstrutiva crônica segundo os critérios do GOLD (Global Iniciative for Chronic Obstrutive Lung Disease, 2018) com fraqueza muscular associada $\left(\mathrm{PI}_{\max }<60 \mathrm{cmH}_{2} \mathrm{O}\right)$ e que preencham os critérios de inclusão do estudo. O protocolo de treinamento utilizado foi baseado no previamente descrito por Charususin e colaboradores (2013).

O recrutamento será realizado no Ambulatório de Pneumologia do Hospital das Clínicas da Universidade Federal de Pernambuco (UFPE), será realizada também divulgação da pesquisa por meio eletrônico e os pacientes que demonstrarem interesse em participar do estudo serão selecionados de acordo com critério de elegibilidade e convidados a participar do estudo. Após o período de divulgação e triagem dos pacientes, será realizado um estudo piloto com 10 pacientes para posterior cálculo do tamanho amostral através dos procedimentos estatísticos adequados.

Os critérios de inclusão serão: Indivíduos de ambos os sexos; Idosos acima de 60 anos; Indivíduos com diagnóstico de DPOC em todos os estádios da doença (I - leve, II- moderada e III - grave); Pacientes que apresentem fraqueza muscular associada $\left(\mathrm{PI}_{\max }<60 \mathrm{cmH}_{2} \mathrm{O}\right)$. Serão excluídos os pacientes que preencherem um ou mais dos seguintes critérios: Indivíduos obesos $\left(\mathrm{IMC} \geq 30 \mathrm{Kg} / \mathrm{m}^{2}\right)$; Indivíduos com doenças reumatológicas ou ortopédicas, deformidades/anormalidades na coluna vertebral ou qualquer outra disfunção anatômica que curse com comprometimento da mecânica do sistema respiratório; Fumantes; Mulheres em período gravídico ou sob suspeita de gravidez; Indivíduos com instabilidade hemodinâmica (frequência cardíaca $>150 \mathrm{bpm}$ ), hipertensão arterial sistêmica (pressão arterial > $149 / 89 \mathrm{mmHg}$ ) ou hipotensão (pressão arterial $<90 / 60 \mathrm{mmHg}$ ) no momento da coleta; Presença de comorbidades 
respiratórias (asma, bronquiectasia, sequela de tuberculose), cardiovasculares; Presença de comorbidades neurológicas ou algum tipo de comprometimento cognitivo que prejudique a compreensão ou execução dos procedimentos atrelados à pesquisa. O estudo será desenvolvido com a participação de dois pesquisadores, o pesquisador A será responsável pela avaliação dos pacientes antes e após a intervenção, enquanto o pesquisador B será responsável pelo acompanhamento dos pacientes na fase de intervenção. O fluxograma de captação e acompanhamento dos pacientes se encontra descrito na figura 1.

A randomização dos indivíduos será realizada por um pesquisador não participante da pesquisa, através do programa www.randomization.com, garantindo o sigilo da alocação. Além disso, para maior confiabilidade, serão utilizados envelopes opacos, lacrados e enumerados sequencialmente para ocultação da alocação. Depois desse processo, os envelopes serão encaminhados ao pesquisador B, responsável pelo acompanhamento dos pacientes durante a fase de intervenção, ficando o pesquisador A cego quanto ao tipo de intervenção que o paciente será submetido, realizando apenas a avaliação das variáveis antes e após a intervenção.

Figura 1. Fluxograma de captação e acompanhamento dos pacientes

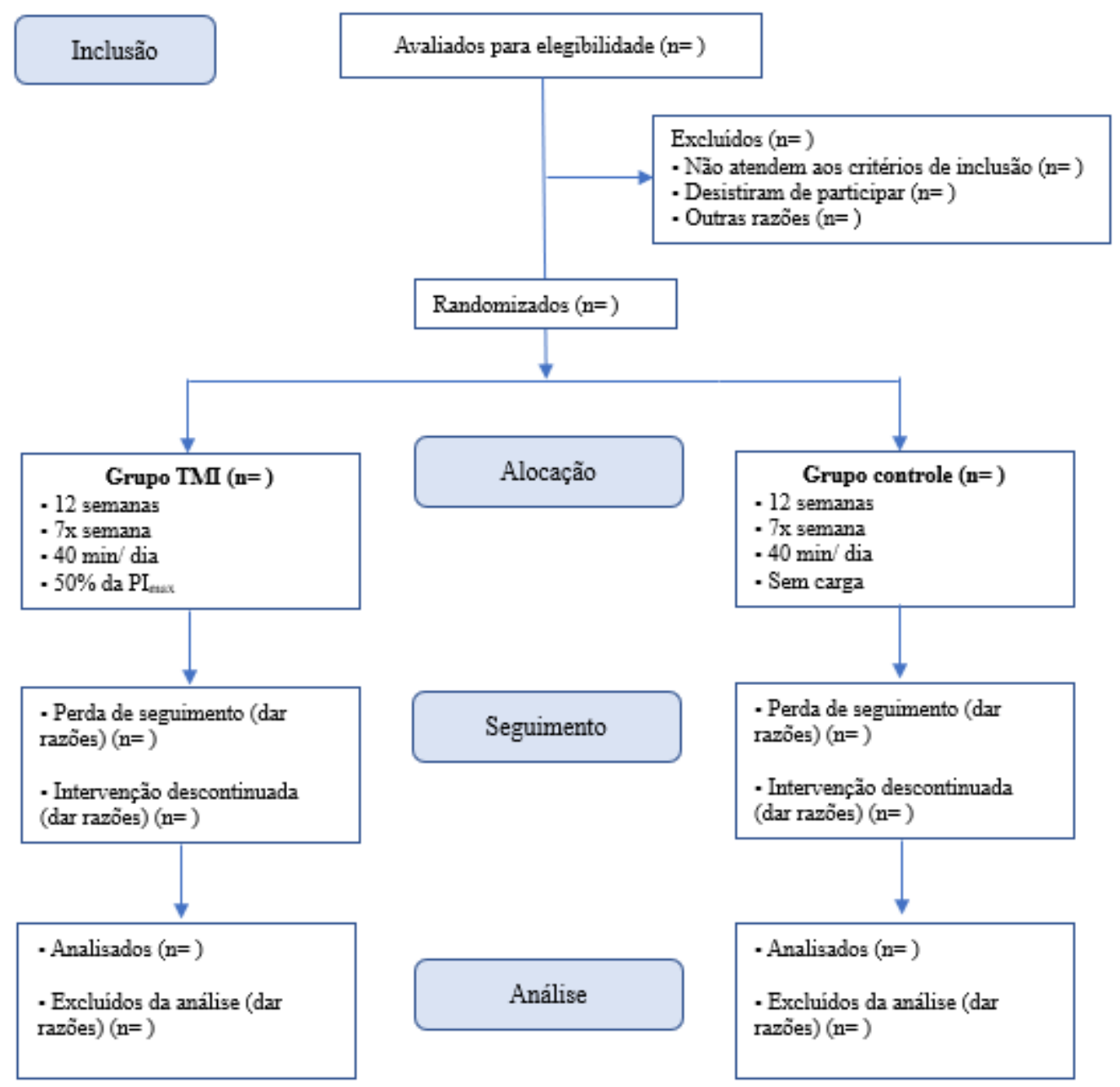




\subsection{Aspectos éticos}

A presente pesquisa atende aos preceitos éticos explicitados na Declaração de Helsinque e seguirá os termos preconizados pelo Conselho Nacional de Saúde (Resolução 196 de 2012) para pesquisa em seres humanos. O projeto de pesquisa será submetido à análise pelo Comitê de Ética em Pesquisa do Centro de Ciências da Saúde da Universidade Federal de Pernambuco. Todos os voluntários serão previamente informados sobre todos os procedimentos da pesquisa bem como seus riscos e benefícios. Ressalta-se que serão incluídos no estudo aqueles indivíduos que concordarem em participar da pesquisa e assinem o Termo de Consentimento Livre e Esclarecido. Fica resguardado o direito de qualquer voluntário recusar-se a participar do estudo, em qualquer momento da coleta dos dados. Os procedimentos que serão utilizados nessa pesquisa não oferecem maiores riscos para os voluntários, e serão prontamente interrompidos na vigência de qualquer relato de desconforto. Aos pacientes que, após a randomização, forem alocados no grupo controle, lhes será dado o direito, ao fim da pesquisa, de participar do programa de Reabilitação Pulmonar da UFPE.

\subsubsection{Conflitos de interesse}

O presente estudo não apresenta qualquer conflito de interesse.

\subsection{Protocolo de treinamento}

O programa de TMI utilizará o dispositivo Power Breathe ${ }^{\circledR}$ durante 8 semanas. O treino será realizado durante 7 dias consecutivos, em cada dia serão realizadas 3 sessões com 2 ciclos cada, totalizando os 6 ciclos diários. Será possibilitado um período de descanso de um (01) minuto entre cada ciclo. Os pacientes do Grupo TMI iniciarão o treinamento com $60 \%$ da $\mathrm{PI}_{\max }$ e a intensidade do treino será aumentada semanalmente em $50 \%$ sob os novos valores de $\mathrm{PI}_{\max }$ mensurados. $\mathrm{O}$ grupo controle realizará o mesmo protocolo de treinamento, porém com $10 \%$ da $\mathrm{PI}_{\max }$ mensurada inicialmente e esse valor se manterá constante ao longo de todo tempo de treinamento. O protocolo descrito foi baseado de Charususin e colaboradores (2013). O acompanhamento desses pacientes se dará tanto pessoalmente, uma vez por semana, para ajustes das cargas do dispositivo como também à distância, por se tratar de um protocolo domiciliar. $\mathrm{O}$ paciente receberá um diário onde relatará sobre a frequência com que executa o treinamento e também será acompanhado através de ligações.

A mensuração das variáveis de força muscular respiratória, volumes e capacidades pulmonares, padrão ventilatório, nível de dispneia, deposição pulmonar e qualidade de vida será realizada antes e após o término do programa de treinamento.

\section{Avaliação Clínica}

Após a triagem inicial e seleção dos pacientes de acordo com os critérios de inclusão estudo, será realizada a avaliação dos dados clínicos, sociodemográficos e antropométricos. A variáveis de massa corporal e estatura serão avaliadas através da balança de bioimpedância elétrica (Biospace ${ }^{\circledR}$ r20, Seoul, Korea). Em seguida será realizado o cálculo do índice de massa corporal (IMC) através da razão entre o peso corporal e estatura elevada ao quadrado $\left(\mathrm{kg} / \mathrm{m}^{2}\right)$. Serão avaliados dados basais referentes à saturação periférica de oxigênio $\left(\mathrm{SpO}_{2}\right)$, frequência cardíaca (FC), pressão arterial sistólica (PAS), pressão arterial diastólica (PAD) e frequência respiratória (FR) através de um monitor multiparamétrico (DIXTAL® 2023 Manaus, Brasil). 


\subsection{Volumes e capacidades pulmonares}

A mensuração dos volumes pulmonares será realizada através da espirometria digital (Micro Medical Microloop MK8®, Rochester, Inglaterra), que é padrão-ouro no que diz respeito à avaliação da função pulmonar, como especificado nas diretrizes da GOLD. O teste será realizado com o indivíduo na posição sentada, com os pés apoiados no chão, coluna ereta e utilização de uma boquilha e clipe nasal. Serão realizadas pelo menos três manobras de capacidade vital forçada (CVF) e de capacidade vital lenta (CVL), respeitando os dois minutos de intervalo entre as manobras, como preconizado pela American Thoracic Society (ATS) (Miler e colaboradores, 2005) e diretrizes de função pulmonar. Será realizada a média das três medidas realizadas e os valores serão expressos em forma de porcentagem do valor predito normal para a população brasileira (Duarte, Pereira \& Rodrigues, 2007) Além das variáveis já mencionadas serão analisadas: a capacidade inspiratória (CI), o $\mathrm{VEF}_{1}$, a relação entre o $\mathrm{VEF}_{1}$ e a capacidade vital forçada ( $\mathrm{VEF}_{1} / \mathrm{CVF}$ ), o pico de fluxo expiratório (PFE) e o fluxo expiratório forçado médio entre $25 \%$ e $75 \%$ da $\mathrm{CVF}\left(\mathrm{FEF}_{25-75 \%}\right.$ ).

\subsection{Plestimografia optoeletrônica}

A plestimografia optoeletrônica (POE) (BTS Bioengineering, Milão, Itália) trata-se de um método indireto e não invasivo de avaliação da variação de volume na caixa torácica, que permite avaliar de forma tridimensional e em tempo real os volumes e suas variações nos três compartimentos que compõem a parede torácica (caixa torácica pulmonar, caixa torácica abdominal e abdome) (Parreira, Vieira, Myrrha, Pessoa, Lage \& Britto, 2012). Serão fixados 89 marcadores reflexivos nas faces ântero-lateral e posterior do tronco em sistema de grade (Figura 2).

Figura 2. Disposição dos 89 marcadores reflexivos para análise da cinemática da parede torácica.
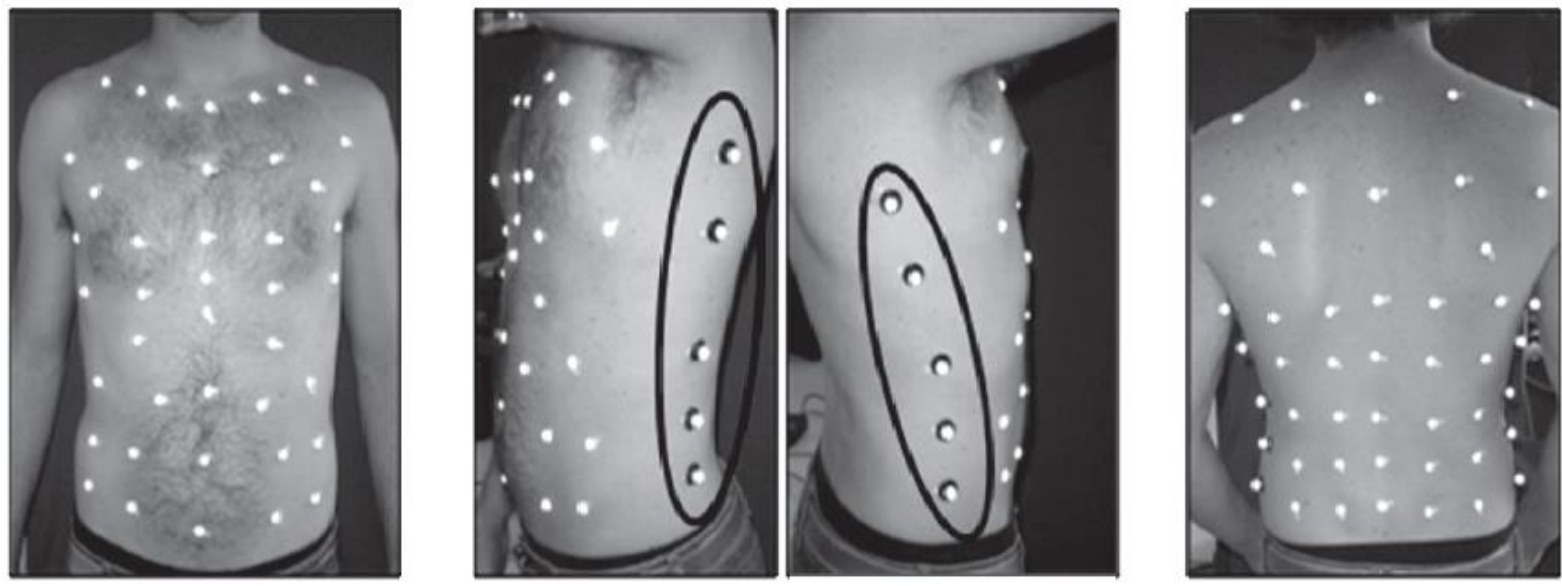

Fonte: Parreira et al. (2012).

\subsection{Força Muscular Respiratória (FMR)}

A força muscular respiratória será mensurada por meio de manovacuometria (Manovacuômetro digital V2.0, NEPEB - UFMG, Minas Gerais, Brasil) (Pessoa e colaboradores, 2014; Ferreira e colaboradores, 2010) em seus componentes de $\mathrm{PI}_{\max }$ e $\mathrm{PE}_{\max }$. A avaliação será realizada com os voluntários sentados, com os pés apoiados no chão, coluna ereta e com uso de boquilha e clipe nasal. Será solicitado que o indivíduo expire até o volume residual (VR) e inspire até a capacidade pulmonar total (CPT), o que resultará na medida da $\mathrm{PI}_{\max }$. Já a $\mathrm{PE}_{\max }$ será avaliada após a inspiração da CPT até o VR. Todas as manobras serão realizadas contra válvula ocluída. Será levado em consideração o maior valor obtido 
dentre as cinco manobras realizadas, contudo estes não podem diferir mais que $10 \%$ do segundo maior valor em ordem decrescente e posteriormente serão comparados com os descritos na literatura (Neder, Andreoni, Lerario, \& Nery,1999).

\subsection{Cintilografia Pulmonar}

Inicialmente os pacientes serão nebulizados com uma mistura de 10 gotas de Brometo de Fenoterol e 20 gotas de Brometo de Ipatrópio diluídos em até $3 \mathrm{ml}$ de soro fisiológico a $0.9 \%$ e associada a uma dose de ácido dietilnotriaminopentacético marcado com tecnécio (DTPA-Tc ${ }^{99 m}$ ) com atividade de $1 \mathrm{mCi} 40$, que será utilizado como marcador para posterior análise da deposição pulmonar através da cintilografia. O procedimento será realizado com o paciente na posição sentada e serão dadas orientações prévias para que os mesmos utilizem a respiração oral de maneira lenta e profunda, realizando uma pausa inspiratória durante 3 segundos e que evite falar para evitar a perda do fármaco. Ao término dessa etapa, os voluntários serão orientados a beber água com o objetivo de remover os resquícios de radioaerossol depositados em regiões de esôfago e orofaringe (França e colaboradores, 2006; Newman, Pitcairn, Hirst \& Rankin, 2003)

Concluída essa etapa, o paciente o paciente será posicionado na posição sentada e previamente orientado a não se movimentar durante a aquisição das imagens. Serão efetuadas a leitura das contagens radioativas na câmara de cintilação de uma cabeça (STARCAM 3200 AC/T GE Medical Systems - U.K.), tais imagens serão armazenadas em matriz 256x256 pixels e serão realizadas nos tempos $0,15,30$ e 60 min, durante 5 minutos em cada tempo. Os arquivos serão armazenados para posterior análise.

$\mathrm{Na}$ análise da deposição de radioaerossol pulmonar, serão delimitadas regiões de interesse (ROI) no sentido transversal: região do terço superior (TS), terço médio (TM) e terço inferior (TI) e no sentido longitudinal: região central (C), intermediária (I) e periférica (P), de acordo com protocolo descrito por Chamberlain e cols (1983) e Graham e colaboradores (1990). Tais ROI'S serão utilizadas para obtenção do cálculo de índice de deposição pulmonar (IDP), obtido através da razão entre a quantidade de contagens de cada ROI pela quantidade total de contagens do respectivo pulmão e expresso em porcentagem. O índice de penetração do radioaerossol (IPR) para cada pulmão será obtido pela razão entre a quantidade de radioatividade presente na região central (C) e a quantidade de radioatividade presente na região periférica (P) (Fauroux e colaboradores, 2000) sendo esta última, a soma na deposição dos ROI intermediário e periférico $(\mathrm{IPR}=\mathrm{C} / \mathrm{P} \times 100)$.

\subsection{Mobilidade e espessura diafragmática}

A ultrassonografia como meio de avaliação trata-se de um recurso não invasivo e livre de radiação, o que constitui algumas das principais vantagens da técnica. A possibilidade de poder correlacionar a análise da mobilidade e espessura diafragmática com a função pulmonar do indivíduo oferece um dado a mais no acompanhamento clínico (Souza e colaboradores,2014), inclusive em pacientes com DPOC (Baria e colaboradores, 2014) O estudo da mobilidade diafragmática será realizada de acordo com o protocolo utilizado no estudo de Testa e colaboradores (2011) com o paciente posicionado decúbito dorsal, em um ângulo de $45^{\circ}$ e avaliará o deslocamento do diafragma desde a CRF até a CPT. Já a avaliação da espessura diafragmática se baseará no utilizado por Souza e colaboradores (2014), realizada com o paciente em decúbito lateral esquerdo e será mensurada em três momentos: na CRF, CPT e manobra de Pimáx. 


\subsection{Nível de dispneia}

Será avaliada através do Medical Research Council Escale Modified (mMRC), uma escala considerada como padrão ouro, proposto pelo GOLD. Nessa escala, os pacientes são estratificados em 4 graus, que vão desde 1 ("normal") até 5 ("muito dispneico para deixar a casa") e se torna possível estabelecer correlação com a qualidade de vida do indivíduo e prognóstico clínico, como será feito nesse estudo, em que a referida escala será aplicada antes e depois da intervenção. Uma outra ferramenta será utilizada para mensurar o estado subjetivo de dispneia dos pacientes quando submetidos ao exercício, trata-se da Escala de Borg Modificada, graduada de 0 (nenhuma dispneia) a 10 (máxima sensação de dispneia) (Kendrick, Baxi \& Smith, 2000).

\subsection{Qualidade de vida}

A QV será avaliada com auxílio do questionário St. George's Respiratory Questionnaire (SGRQ), específico para pacientes com DPOC (Ferrer e colaboradores, 2002). Ele é composto por 76 itens divididos em 3 domínios (sintomas, atividades e impacto da doença), em cada domínio os itens são codificados e transformados em escala de zero (pior QV) a 100 pontos (melhor QV). Outra ferramenta que muito se relaciona com a anterior também será utilizada, se trata do COPD Assesment Test (CAT), que avalia o estado de saúde e o impacto sintomático da DPOC na vida dos pacientes (Lozano e colaboradores, 2010)

\subsection{Capacidade funcional}

Será avaliada através do Questionário Internacional de Atividade Física (IPAQ), ferramenta disponível na língua portuguesa, nas versões curta e longa e que tem a finalidade de estimar o nível de atividade física através de diferentes populações. Essa mensuração se dá através de informações a respeito da frequência e duração de atividades como caminhadas ou atividades cotidianas realizadas durante a semana, ou aquelas de intensidade moderada ou vigorosa (Guedes, Correa, Elisabete \& Pinto, 2005).

Outro método de avaliar a capacidade funcional do paciente com DPOC será através do Teste de Caminhada de 6 Minutos (TC6M), um teste simples e prático de ser aplicado, que permite avaliar a resposta dos sistemas pulmonar e cardiovascular, como por exemplo, quando submetido ao exercício (ATS statement: guidelines for the six-minute walk test. 2002).

\section{Análise de Dados}

Os dados coletados serão acondicionados em uma ficha de avaliação e posteriormente digitalizados em um banco de dados específico no Microsoft Excel® (Versão 2016). A análise dos dados será realizada através do software Statistical Package for Social Science versão 20.0. Os dados serão expressos em frequência para as variáveis categóricas e em medidas de tendência central e de dispersão para as variáveis numéricas, expressos em média e desvio padrão ou mediana e intervalo interquartil A normalidade das variáveis analisadas será testada através do teste de Kolmogorov-Smirnov e a homogeneidade da amostra será avaliada através do teste de Levene. O teste t Student independente ou teste U de MannWhithey será utilizado para comparação entre os grupos no que diz respeito aos dados clínicos, sociodemográficos, antropométricos, percepção de dispneia volumes e capacidade pulmonares, força muscular respiratória, deposição pulmonar do radioaerossol, espessura e mobilidade diafragmática e qualidade de vida. Todos os testes serão aplicados admitindo-se um nível de confiança de $95 \%$, considerando um valor de $\mathrm{p}$ como significativo quando $<0,05$. 


\section{Considerações Finais}

Acreditamos que se trata de um estudo inédito pois até o momento não encontramos registros com as intervenções propostas sob a população em questão. Consideramos que a alta prevalência dessa patologia, os índices de exacerbação e hospitalização desses indivíduos justifiquem a necessidade dessa gama de avaliações e análises com as ferramentas aqui descritas, estas que poderão fornecer o maior número de evidências possíveis sobre pacientes que convivem com essa doença crônica, levando a um melhor manejo destes no futuro. A decisão de publicar esse protocolo vem da ideia de que a ciência não pode ser monopolizada e sim compartilhada, beneficiando assim o máximo de pessoas e possibilitando que esse trabalho possa ser replicado, trazendo novas ideias e desfechos, esse é o nosso maior objetivo e acredito que foi alcançado com a elaboração desse protocolo.

Para os trabalhos futuros uma sugestão interessante seria incluir pacientes com diagnóstico de DPOC e que tiveram COVID. Poderiam ser estudados em dois grupos: os que tiveram casos leves de DPOC e não precisaram de internamento hospitalar e os que tiveram casos mais graves que levaram a necessidade de internamento e uso de oxigenoterapia e ventilação não-invasiva, por exemplo. Acreditamos aplicar o protocolo aqui descrito e avaliar os dados desses pacientes em um período pós-COVID imediato com que descrevemos resultaria em um ou mais estudos de grande relevância e contribuição para o meio acadêmico.

\section{Referências}

ATS Committee on Proficiency Standards for Clinical Pulmonary Function Laboratories (2002). ATS statement: guidelines for the six-minute walk test. American journal of respiratory and critical care medicine, 166(1), 111-117. doi:10.1164/ajrccm.166.1.at1102

Baria, M. R., Shahgholi, L., Sorenson, E. J., Harper, C. J., Lim, K. G., Strommen, J. A. et al. (2014). B-mode ultrasound assessment of diaphragm structure and function in patients with COPD. Chest. 146(3): 680-5. doi: 10.1378/chest.13-2306

Barreiro, E., Bustamante,V., Cejudo, P., Gáldiz, J.B., Gea, J., de Lucas P., et al (2015). Normativa SEPAR sobre disfunción muscular de los pacientes con enfermedad pulmonar obstructiva crónica. Arch Bronconeumol. 51(8):384-95. doi:10.1016/j.arbres.2015.04.011

Beckerman, M., Magadle, R., Weiner, M., \& Weiner, P. (2005). The effects of 1 year of specific inspiratory muscle training in patients with COPD. Chest, 128(5), 3177-3182. doi:10.1378/chest.128.5.3177

Chamberlain, M. J., Morgan, W. K., \& Vinitski, S. (1983). Factors influencing the regional deposition of inhaled particles in man. Clinical science (London, England : 1979).64(1), 69-78. doi:10.1042/cs0640069

Charususin, N., Gosselink, R., Decramer, M., McConnell, A., Saey, D., Maltais, F., Derom, E., Vermeersch, S., van Helvoort, H., Heijdra, Y., Klaassen, M., Glöckl, R., Kenn, K., \& Langer, D. (2013). Inspiratory muscle training protocol for patients with chronic obstructive pulmonary disease (IMTCO study): a multicentre randomised controlled trial. BMJ open. 3(8), e003101. doi:10.1136/bmjopen-2013-003101

Chen, H., Dukes, R., \& Martin, B. J. (1985). Inspiratory muscle training in patients with chronic obstructive pulmonary disease. The American review of respiratory disease. 131(2), 251-255. doi:10.1164/arrd.1985.131.2.251

DiBonaventura, M. d., Paulose-Ram, R., Su, J., McDonald, M., Zou, K. H., Wagner, J. S., \& Shah, H. (2012). The burden of chronic obstructive pulmonary disease among employed adults. International journal of chronic obstructive pulmonary disease, 7, 211-219. doi:10.2147/COPD.S29280

Duarte AA de O, Pereira CA de C, Rodrigues SCS. (2007).Validação de novos valores previstos brasileiros para a espirometria forçada na raça branca e comparação com os valores previstos obtidos por outras equações de referência. J Bras Pneumol.33(5):527-35.

Fauroux, B., Itti, E., Pigeot, J., Isabey, D., Meignan, M., Ferry, G., Lofaso, F., Willemot, J. M., Clément, A., \& Harf, A. (2000). Optimization of aerosol deposition by pressure support in children with cystic fibrosis: an experimental and clinical study. American journal of respiratory and critical care medicine, 162(6), 2265-2271. doi:10.1164/ajrccm.162.6.2003069

Ferreira, J. L., Pereira, N.C., Oliveira, Jr. M., Vasconcelos, F. H., Parreira, V. F. \& Tierra-Criollo, C. J. (2010). Maximum respiratory pressure measuring system: calibration and evaluation of uncertainty. Sba Control Automação Soc Bras Autom. 21(6):588-97. doi:10.1590/S0103-17592010000600004

Ferrer, M., Villasante, C., Alonso, J., Sobradillo, V., Gabriel, R., Vilagut, G., Masa, J. F., Viejo, J. L., Jiménez-Ruiz, C. A., \& Miravitlles, M. (2002). Interpretation of quality of life scores from the St George's Respiratory Questionnaire. The European respiratory journal, 19(3), 405-413. doi:10.1183/09031936.02.00213202

França, E. E. T., Dornelas de Andrade, A. F., Cabral, G., Almeida-Filho, P., Silva, K. C., Galindo-Filho, V. C., et al. (2006). Nebulization associated with Bi-level noninvasive ventilation: Analysis of pulmonary radioaerosol deposition. Respir Med. 100(4):721-8. doi: 10.1016/j.rmed.2005.07.012 
Goldstein, R., De Rosie, J., Long, S., Dolmage, T., \& Avendano, M. A. (1989). Applicability of a threshold loading device for inspiratory muscle testing and training in patients with COPD. Chest, 96(3), 564-571. doi:10.1378/chest.96.3.564

Gosselink, R., De Vos, J., van den Heuvel, S. P., Segers, J., Decramer, M., \& Kwakkel, G. (2011). Impact of inspiratory muscle training in patients with COPD: what is the evidence?.The European respiratory journal, 37(2), 416-425. doi:10.1183/09031936.00031810

Graham, D. R., Chamberlain, M. J., Hutton, L., King, M., \& Morgan, W. K. (1990). Inhaled particle deposition and body habitus. British journal of industrial medicine, 47(1), 38-43. doi:10.1136/oem.47.1.38

Gruenberger, J. B., Vietri, J., Keininger, D. L., \& Mahler, D. A. (2017). Greater dyspnea is associated with lower health-related quality of life among European patients with COPD. International journal of chronic obstructive pulmonary disease. 12, 937-944. doi:10.2147/COPD.S123744

Guedes D. P, Correa C., Elisabete J., Pinto R. (2005). Reprodutibilidade e validade do Questionário Internacional de Atividade Física em adolescentes. Rev Bras Med Esporte. 11:151-8. doi: 10.1590/S1517-86922005000200011

Hamilton, A. L., Killian, K. J., Summers, E., \& Jones, N. L. (1995). Muscle strength, symptom intensity, and exercise capacity in patients with cardiorespiratory disorders. American journal of respiratory and critical care medicine, 152(6 Pt 1), 2021-2031. doi:10.1164/ajrccm.152.6.8520771

Geddes, E. L., O'Brien, K., Reid, W. D., Brooks, D., \& Crowe, J. (2008). Inspiratory muscle training in adults with chronic obstructive pulmonary disease: an update of a systematic review. Respiratory medicine, 102(12), 1715-1729. doi:10.1016/j.rmed.2008.07.005

Hu, J., \& Meek, P. (2005). Health-related quality of life in individuals with chronic obstructive pulmonary disease. Heart \& lung: the journal of critical care, 34(6), 415-422. doi:10.1016/j.hrtlng.2005.03.008

Kendrick, K. R., Baxi, S. C., \& Smith, R. M. (2000). Usefulness of the modified 0-10 Borg scale in assessing the degree of dyspnea in patients with COPD and asthma. Journal of emergency nursing, 26(3), 216-222. doi:10.1016/s0099-1767(00)90093-X

Killian, K. J., \& Jones, N. L. (1988). Respiratory muscles and dyspnea. Clinics in chest medicine, 9(2), 237-248.Disponível em: https://pubmed.ncbi.nlm.nih.gov/3292125/

Lanza, F., de Camargo, A. A., Archija, L. R., Selman, J. P., Malaguti, C., \& Dal Corso, S. (2013). Chest wall mobility is related to respiratory muscle strength and lung volumes in healthy subjects. Respiratory care, 58(12), 2107-2112. doi: 10.4187/respcare.02415

Leith, D. E., \& Bradley, M. (1976). Ventilatory muscle strength and endurance training. Journal of applied physiology, $41(4)$, 508-516. doi:10.1152/jappl.1976.41.4.508

Lozano, R., Naghavi, M., Foreman, K., Lim, S., Shibuya, K., Aboyans, V., Abraham, J. et al. (2012). Global and regional mortality from 235 causes of death for 20 age groups in 1990 and 2010: a systematic analysis for the Global Burden of Disease Study 2010. Lancet (London, England), 380(9859), 2095-2128. doi:10.1016/S0140-6736(12)61728-0

Martínez-Llorens J., Ausín P., Roig A., Balañá A., Admetlló M., Muñoz L, et al. (2011) Presión inspiratoria nasal: ¿una alternativa para la evaluación de la fuerza muscular inspiratoria? Arch Bronconeumol. 47(4):169-75. doi: 10.1016/j.arbres.2011.01.002

McCarthy, B., Casey, D., Devane, D., Murphy, K., Murphy, E., \& Lacasse, Y. (2015). Pulmonary rehabilitation for chronic obstructive pulmonary disease. The Cochrane database of systematic reviews, (2), CD003793. doi:10.1002/14651858.CD003793.pub3

Miller, M. R., Crapo, R., Hankinson, J., Brusasco, V., Burgos, F., Casaburi, R., Coates, A., Enright, P., van der Grinten, C. P., Gustafsson, P., Jensen, R., Johnson, D. C., MacIntyre, N., McKay, R., Navajas, D., Pedersen, O. F., Pellegrino, R., Viegi, G., Wanger, J., \& ATS/ERS Task Force (2005). General considerations for lung function testing. The European respiratory journal, 26(1), 153-161. doi: 10.1183/09031936.05.00034505

Neder, J. A., Andreoni, S., Lerario, M. C., \& Nery, L. E. (1999). Reference values for lung function tests. II. Maximal respiratory pressures and voluntary ventilation. Brazilian journal of medical and biological research = Revista brasileira de pesquisas medicas e biologicas, 32(6), 719-727. doi: $10.1590 / \mathrm{s} 0100-879 \times 1999000600007$

Parreira, V. F., Vieira, D. S. R, Myrrha, M. A. C, Pessoa, I. M. B. S, Lage, S. M., Britto, R. R.(2012). Pletismografia optoeletrônica: uma revisão da literatura Optoelectronic plethysmography: a review of the literature.Rev Bras Fisioter.1616(66):439-53. Disponível em: https://www.scielo.br/j/rbfis/a/xBrSywjG7RN8tjvFLzJC9NR/?format=pdf\&lang=pt

Pessoa, I. M. B. S., Pereira, H. L. A., Aguiar, L. T., Tagliaferri, T, L., Silva L. A. M da, Parreira, V. F. (2014). Test-retest reliability and concurrent validity of a digital manovacuometer. Fisioter e Pesquisa. 2014;6627-31270. doi: 10.590/1809-2950/63521032014

Petrovic, M., Reiter, M., Zipko, H., Pohl, W., \& Wanke, T. (2012). Effects of inspiratory muscle training on dynamic hyperinflation in patients with COPD. International journal of chronic obstructive pulmonary disease, 7, 797-805. doi:10.2147/COPD.S23784

Polkey, M. I., Kyroussis, D., Hamnegard, C. H., Mills, G. H., Green, M., \& Moxham, J. (1996). Diaphragm strength in chronic obstructive pulmonary disease. American journal of respiratory and critical care medicine, 154(5), 1310-1317. doi: 10.1164/ajrccm.154.5.8912741

Souza, H., Rocha, T., Pessoa, M., Rattes, C., Brandão, D., Fregonezi, G., Campos, S., Aliverti, A., \& Dornelas, A. (2014). Effects of inspiratory muscle training in elderly women on respiratory muscle strength, diaphragm thickness and mobility. The journals of gerontology. Series A, Biological sciences and medical sciences, 69(12), 1545-1553. doi:10.1093/gerona/glu182

Testa, A., Soldati, G., Giannuzzi, R., Berardi, S., Portale, G., Gentiloni, S. N. (2011). Ultrasound M-Mode assessment of diaphragmatic kinetics by anterior transverse scanning in healthy subjects. Ultrasound Med Biol.37(1):44-52. doi:10.1016/j.ultrasmedbio.2010.10.004 
Research, Society and Development, v. 11, n. 3, e33211326707, 2022

(CC BY 4.0) | ISSN 2525-3409 | DOI: http://dx.doi.org/10.33448/rsd-v11i3.26707

Vilaró, J., Ramirez-Sarmiento, A., Martínez-Llorens, J. M., Mendoza, T., Alvarez, M., Sánchez-Cayado, N., Vega, A., et al. (2010). Global muscle dysfunction as a risk factor of readmission to hospital due to COPD exacerbations. Respiratory medicine, 104(12), $1896-1902$. doi:10.1016/j.rmed.2010.05.001

Vogelmeier, C. F., Criner, G. J., Martinez, F. J., Anzueto, A., Barnes, P. J., Bourbeau, J., Celli, B. R., et al. (2017). Global Strategy for the Diagnosis, Management, and Prevention of Chronic Obstructive Lung Disease 2017 Report. GOLD Executive Summary. American journal of respiratory and critical care medicine, 195(5), 557-582. doi:10.1164/rccm.201701-0218PP

Wieshammer, S., \& Dreyhaupt, J. (2008). Dry powder inhalers: which factors determine the frequency of handling errors?.Respiration; international review of thoracic diseases, 75(1), 18-25. doi: 10.1159/000109374 